\title{
Management of Hospitalized Adult Patients with Coronavirus 2019 (COVID-19): A Therapeutic Guideline
}

\section{Pir Hossein Kolivand ${ }^{1 *}$, Vahid Dehestani ${ }^{1}$, Hadi Kazemi ${ }^{2}$}

${ }^{1}$ Shefa Neuroscience Research Center, Khatam Alanbia Hospital, Tehran, Iran

${ }^{2}$ Pediatric Department, Medical Faculty, Shahed University, Tehran, Iran

\section{A BSTRACT}

Introduction: COVID-19, caused by the SARS-CoV-2 strain, is highly contagious and can be fatal in acute cases. In severe cases, hypoxia leads to hypoxemia. In these conditions, oxygen management using ventilation and intubation is very necessary. Optimizing and standardizing treatment management through clinical data can improve the quality of care as well as the patient's condition. Materials and Methods: In this study, using data from randomized clinical trials and systematic review and meta-analysis, several critical PICOs (population, intervention, comparator, and outcome) were collected. The GRADE approach was also used to evaluate the quality of evidence and the effectiveness of the recommendations derived from the studies. Results: Based on the available evidence, the following recommendations advised to improve the condition of patients: I) the use of systemic corticosteroids in patients who need oxygen and the use of anticoagulant drugs during hospitalization recommended. II) Treatment of monoclonal antibody (i.e., interleukin -6-receptor antagonist) with oxygen is conditionally recommended in patients with respiratory failure. III) The hydroxychloroquine in combination with lopinavir-ritonavir should not be used in patients with respiratory failure. IV) The use of azithromycin, hydroxychloroquine in combination with azithromycin, colchicine, and remdesivir are recommended temporarily in patients with ventilation. V) Early diagnosis and initiation of treatment have a significant effect on the patient's recovery. Conclusion: The proposed guideline obtained from the evaluation of clinical trials, systematic reviews, and meta-analyses can improve the outcomes of patients with COVID-19 disease.

Keywords:

1. COVID-19

2. Anticoagulants

3. Interleukins

*Corresponding Author: Pir Hossein Kolivand

Email:peirhossein@yahoo.com 
مديريت درمان بيماران بزركسال بسترى شده به علت ابتلا به كرونا ويروس 9 إب (COVID-19): يك كايدلاين درمانى

\author{
"يير حسين كوليوند"'، وحيد دهستانى'، هادى كاظمى'
}

'مركز تحقيقات علوم اعصاب شفاك، بيمارستان خاتم الانبياء، تهران، ايران

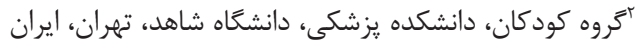

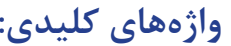

19- 19 r ب

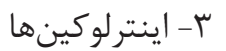

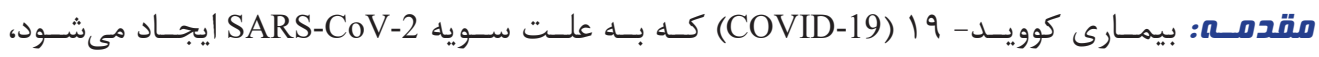

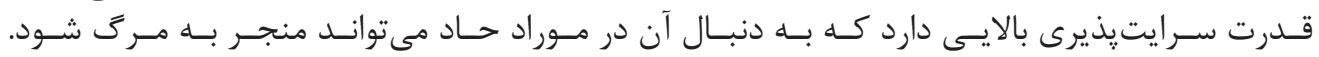

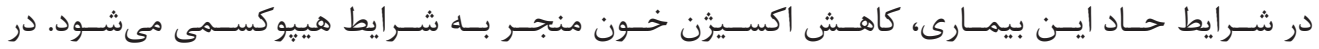

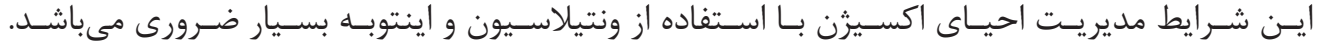

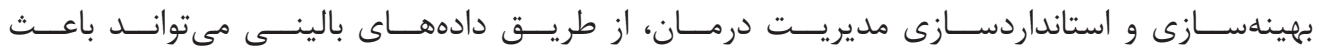

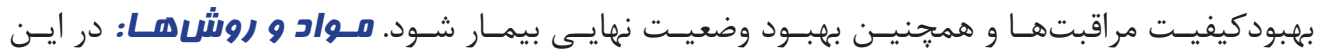

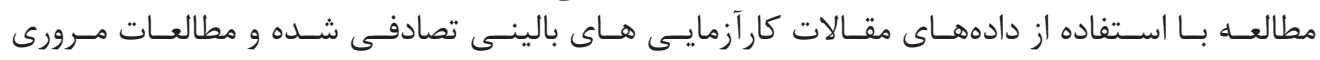

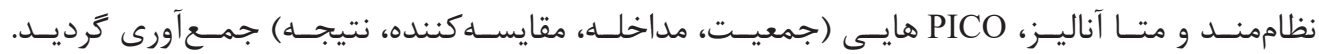

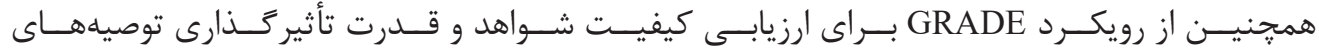

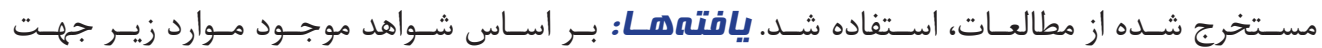

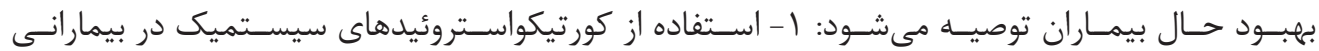

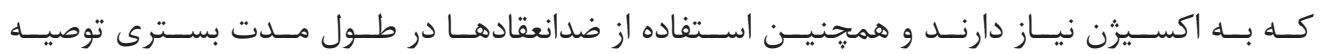

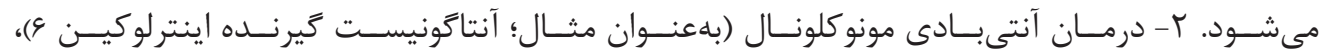

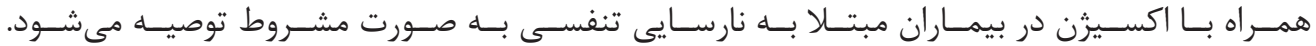

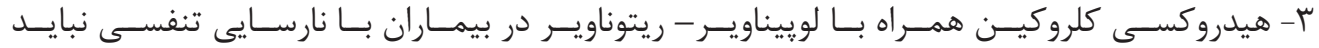

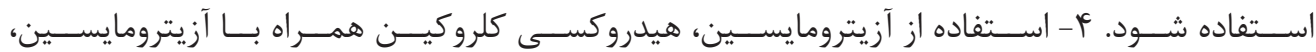

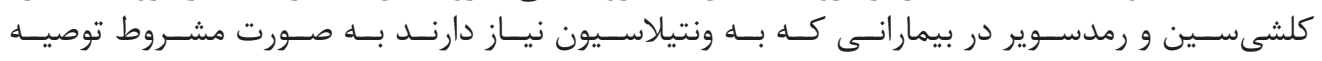

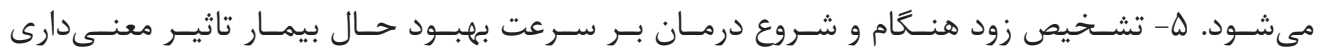

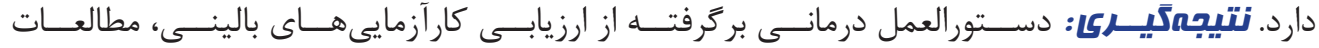

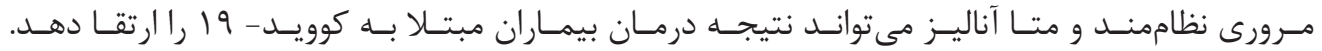

$$
\text { *ويسنده مسئول: پيرحسين كوليوند }
$$




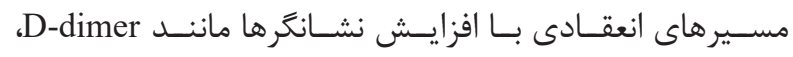

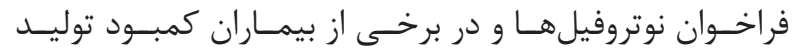

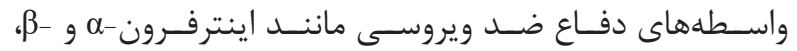

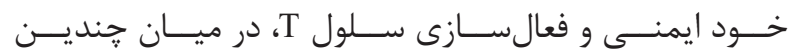

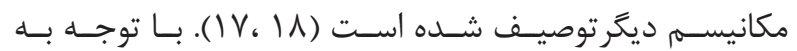

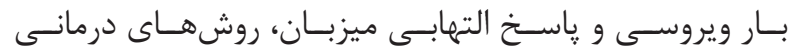

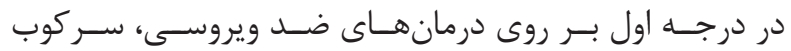

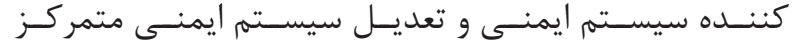

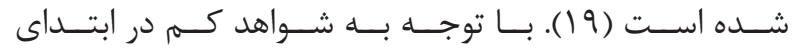

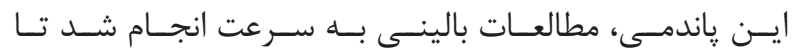

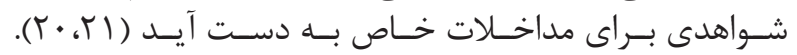

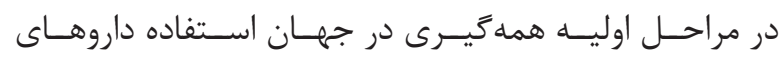

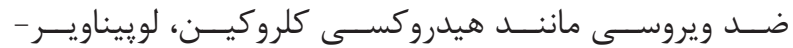

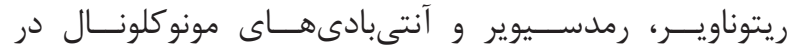

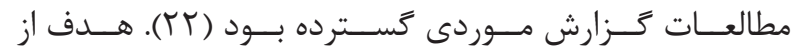

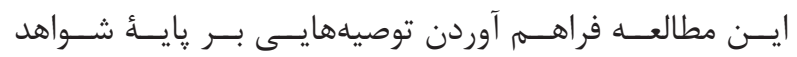

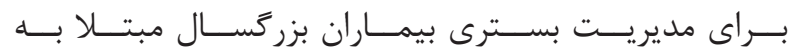

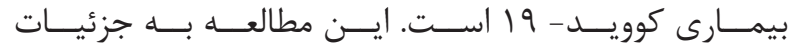

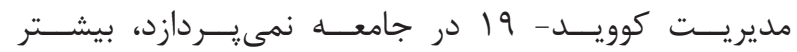

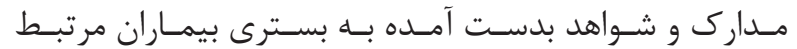

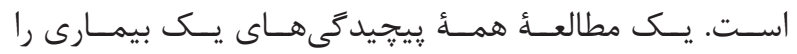

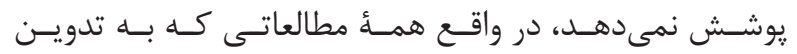

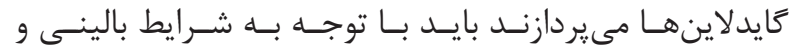

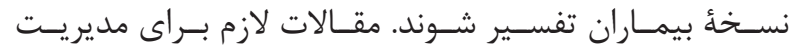

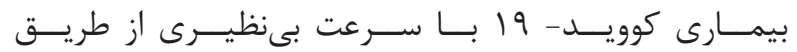

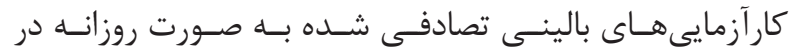

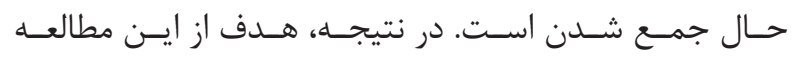

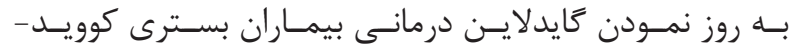

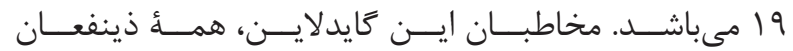

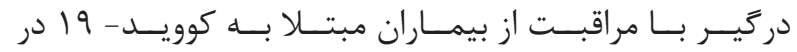

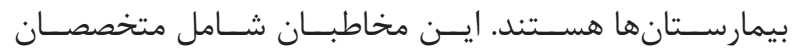

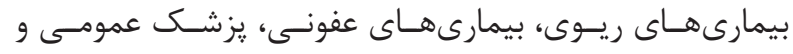

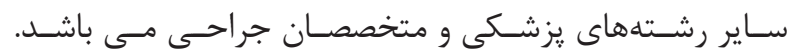

\section{مواد و روشها - - ماد}

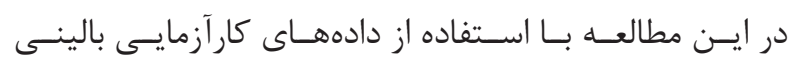

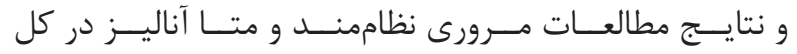

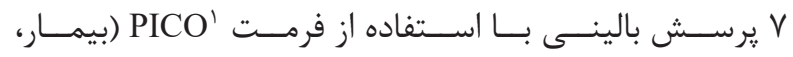

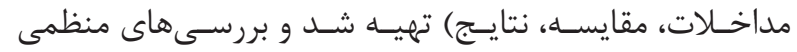

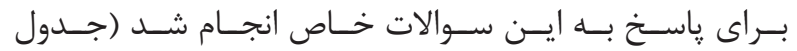

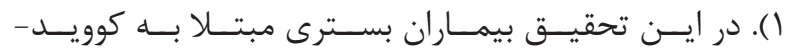

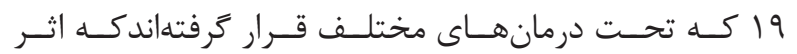

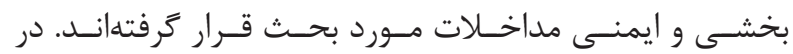

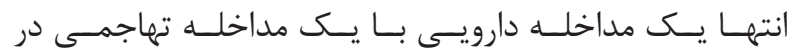

مقدمه

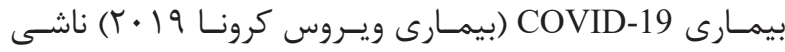

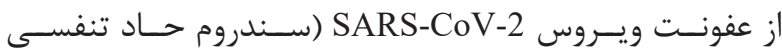

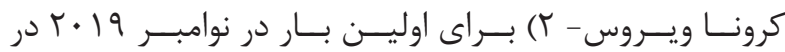

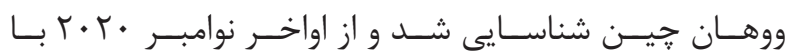

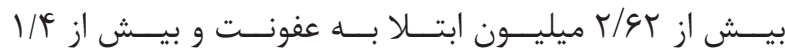

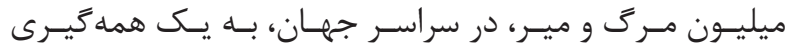

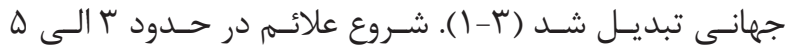

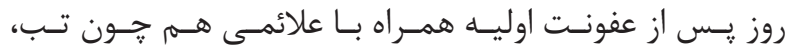

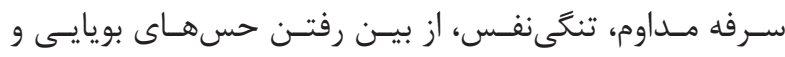

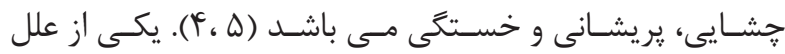

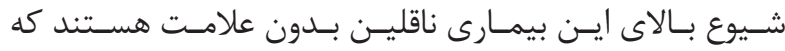

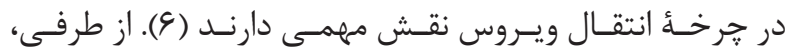

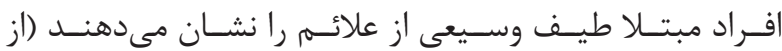

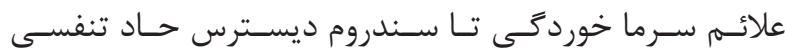

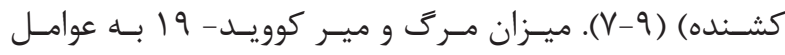

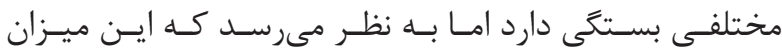

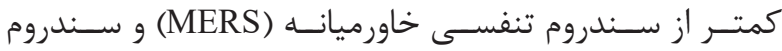

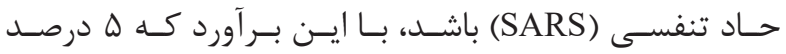

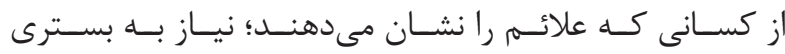

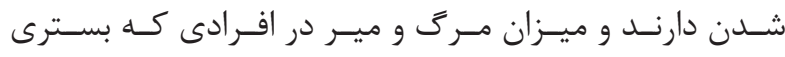

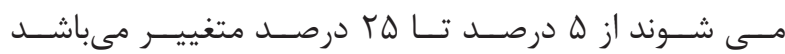

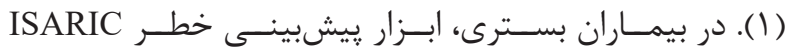

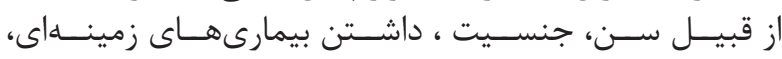

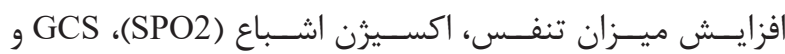

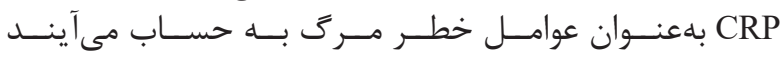

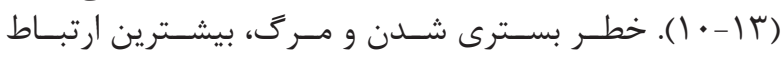

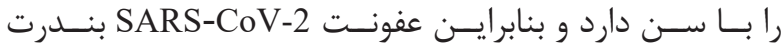

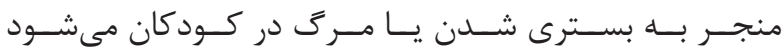

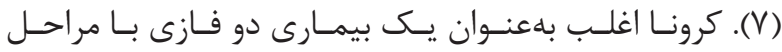

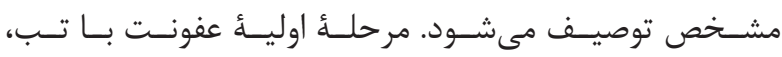

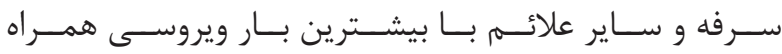

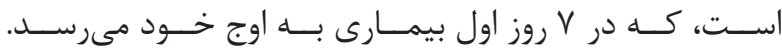

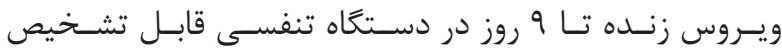

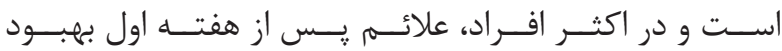

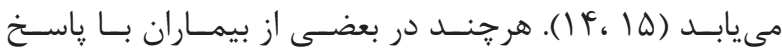

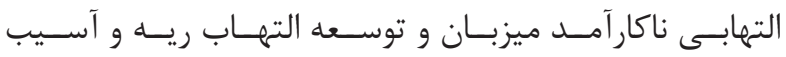

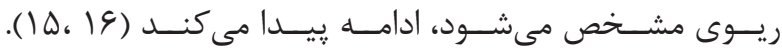

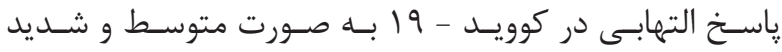

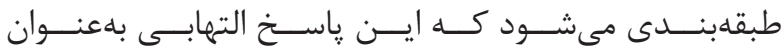

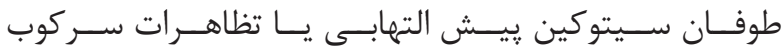

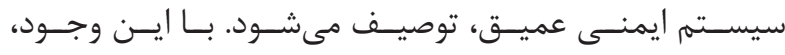

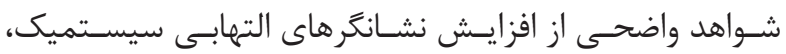

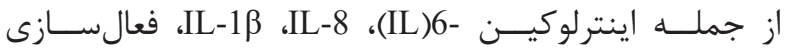




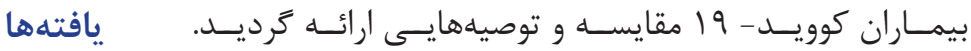

جدول ا- تدوين مداخلات مختلف در قالب PICO

\begin{tabular}{|c|c|c|c|}
\hline نتيجه & 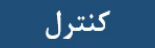 & 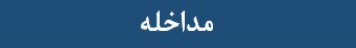 & جمعيت - \\
\hline مرق و مير & مراقبت معمول & كورتيكواستروئيدها & بيماران بسترى كوويد- 19 \\
\hline مرق و مير & مراقبت معمول & آنتى بادىهاى مونوكلونال & بيماران بسترى كوويد- 19 \\
\hline مرى و مير & مراقبت معمول & هيدروكسى كلروكين & بيماران بسترى كوويد- 19 \\
\hline ترخيص از بيمارستان & مراقبت معمول & آزيترومايسين & بيماران بسترى كوويد- 19 \\
\hline اكسيرن اشباع خون & مراقبت معمول & هيدروكسى كلروكين و آزيترومايسين & بيماران بسترى كوويد- 19 \\
\hline ترخيص از بيمارستان و مرك و مير & مراقبت معمول & 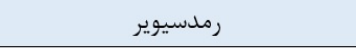 & بيماران بسترى كوويد- 19 \\
\hline 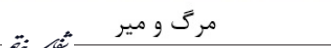 & مراقبت معمول & اينترفرون بتا & بيماران بسترى كوويد- 19 \\
\hline
\end{tabular}

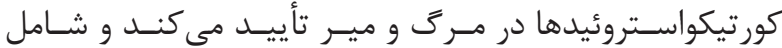

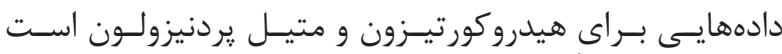

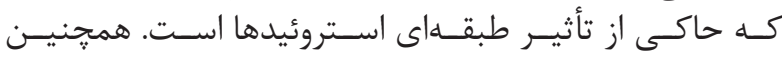

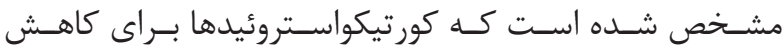

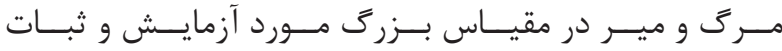

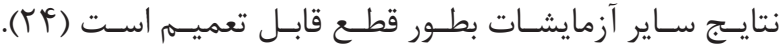

\section{קֵيكوץ}

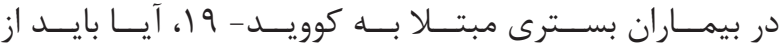

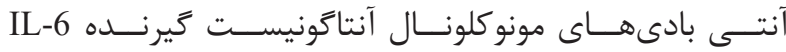

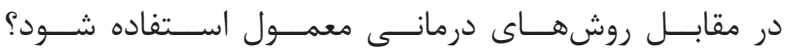

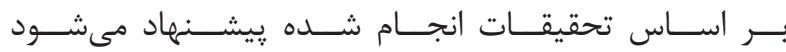

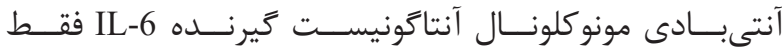

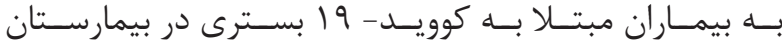

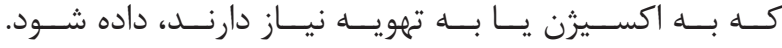

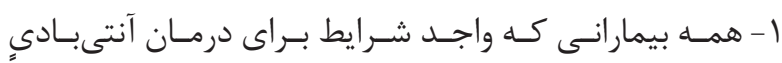

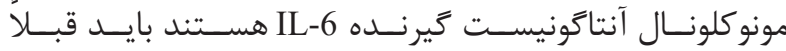

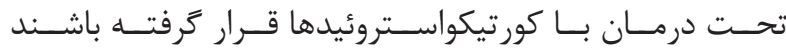

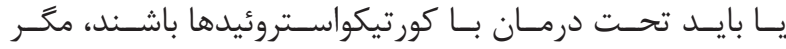

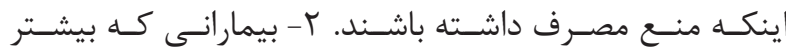

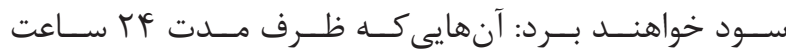

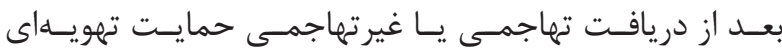

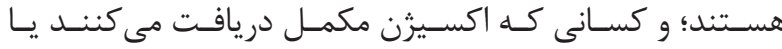

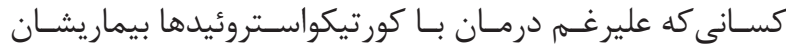

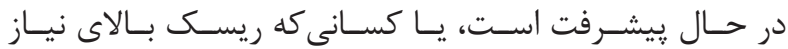

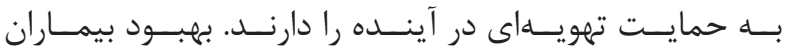

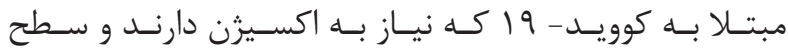

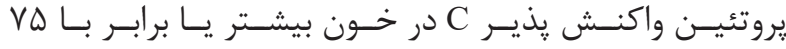

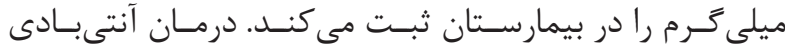

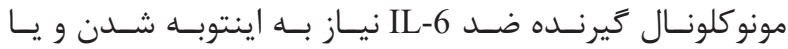

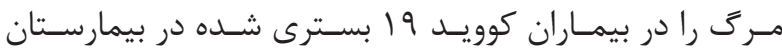

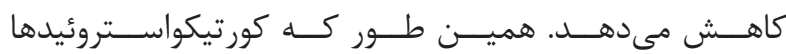

بيكو 1

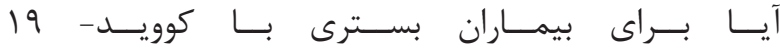

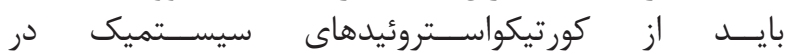

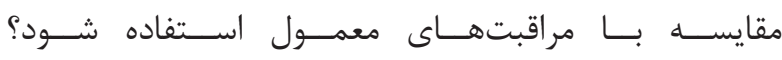

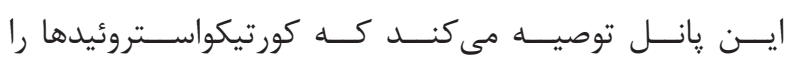

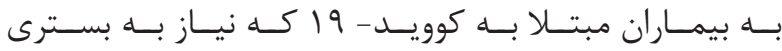

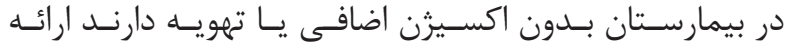

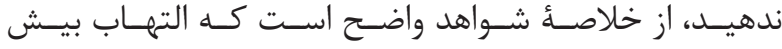

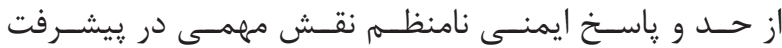

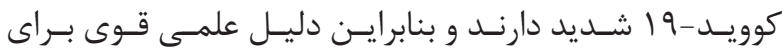

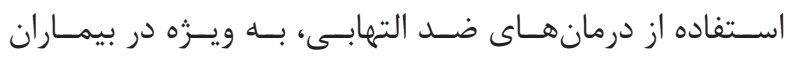

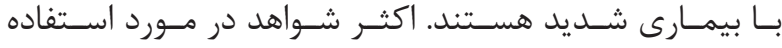

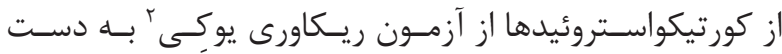

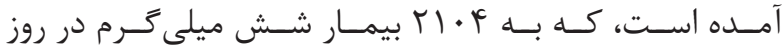

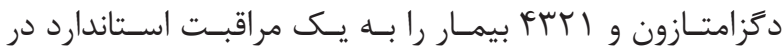

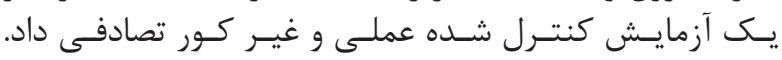

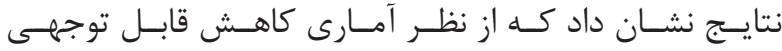

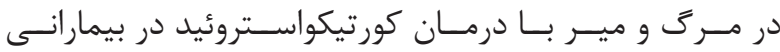

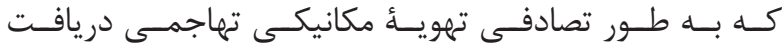

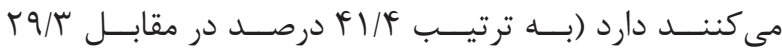

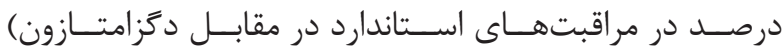

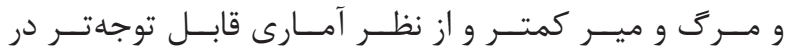

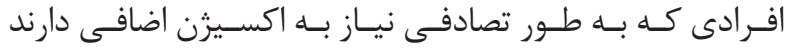

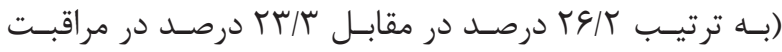

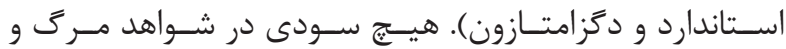

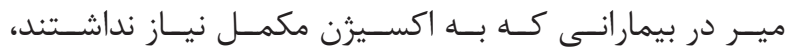

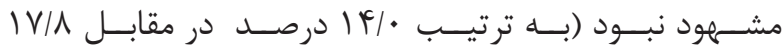

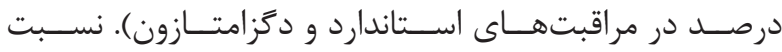

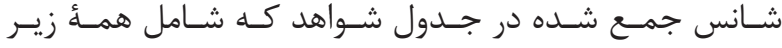

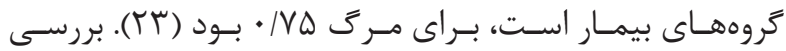

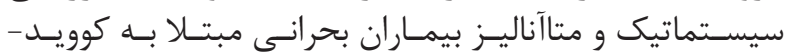

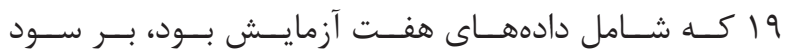




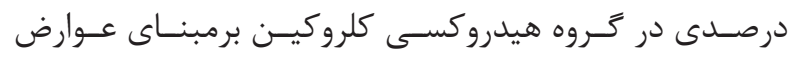

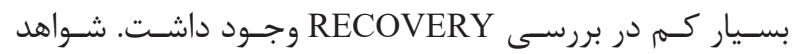

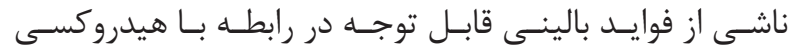

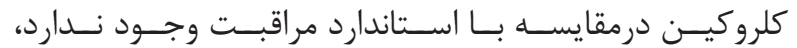

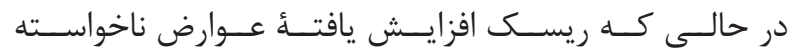

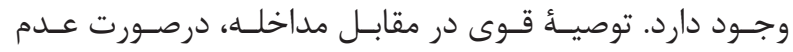

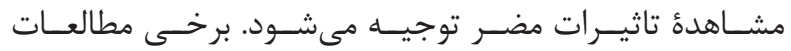

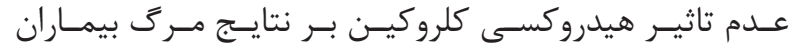

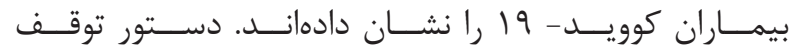

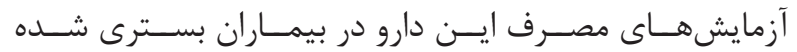

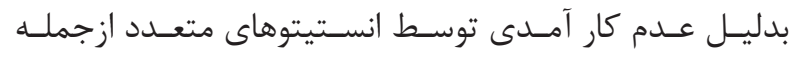

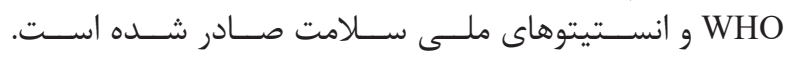

\section{קِيكو}

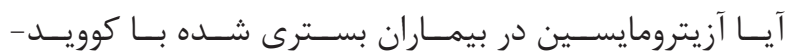

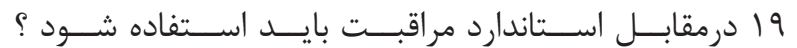

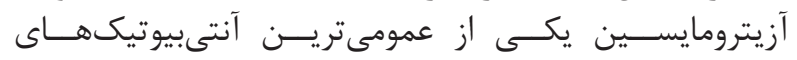

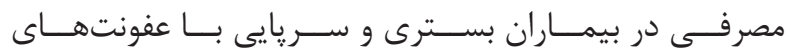

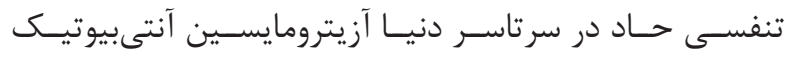

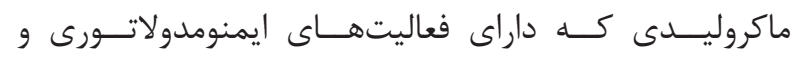

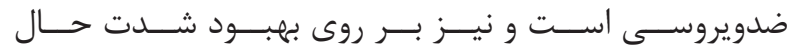

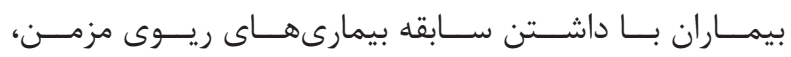

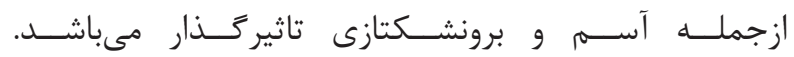

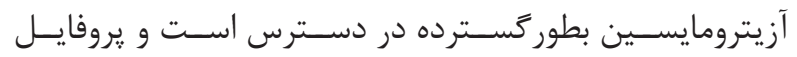

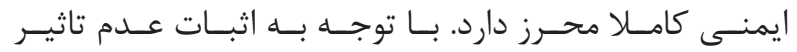

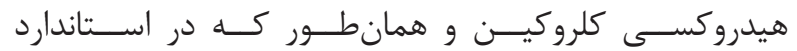

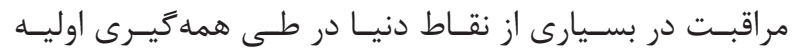

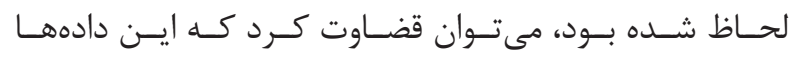

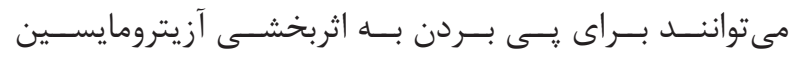

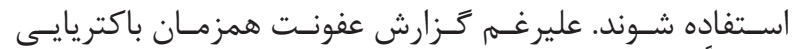

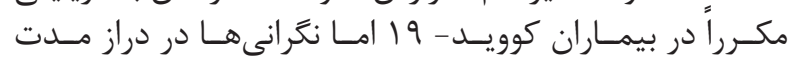

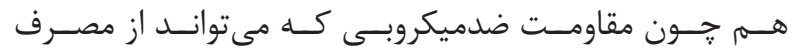

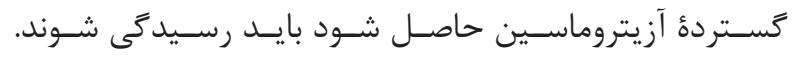

\section{هيكو ه}

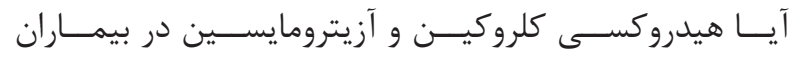

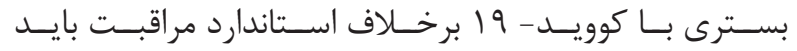

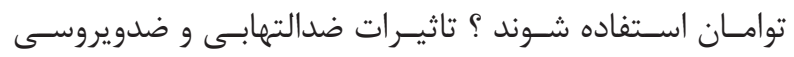

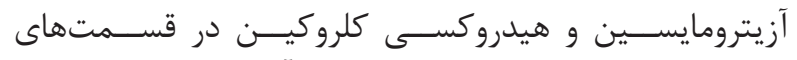

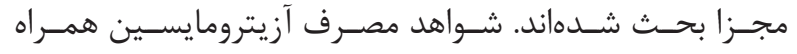

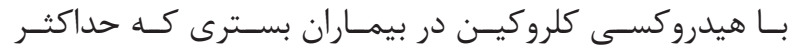

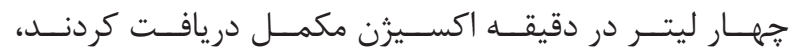

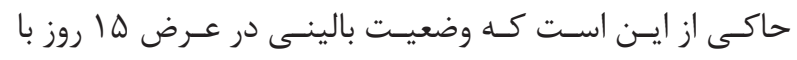

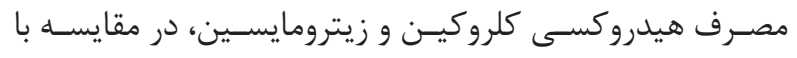

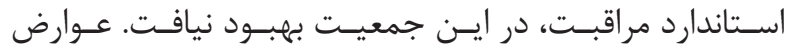

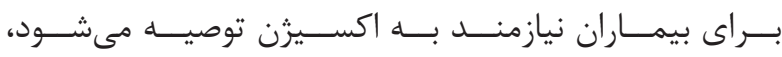

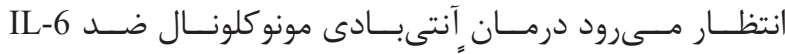

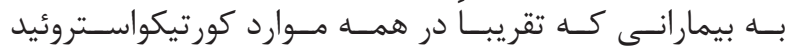

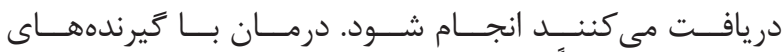

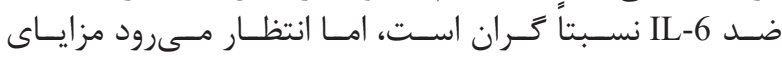

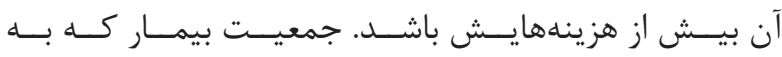

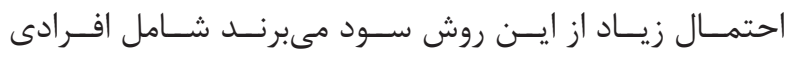

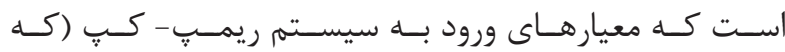

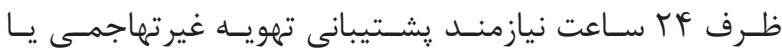

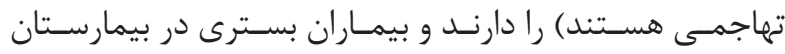

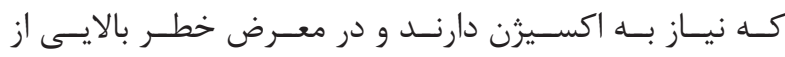

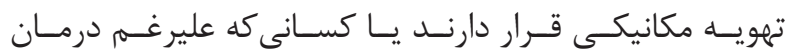

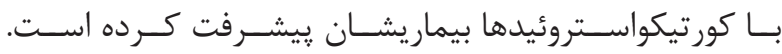

קֵيكوץ

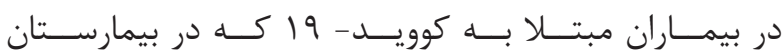

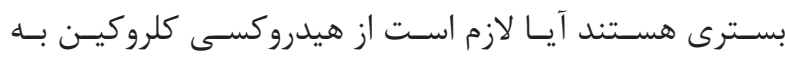

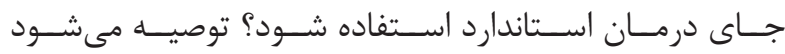

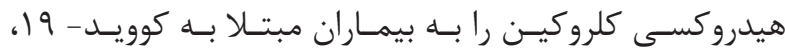

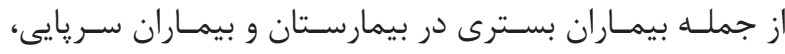

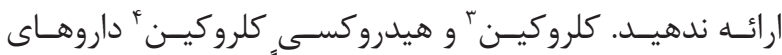

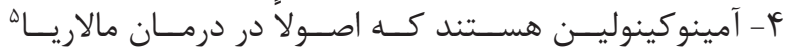

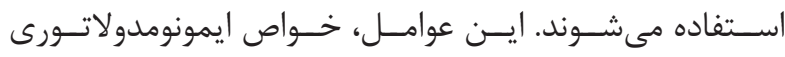

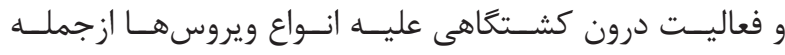

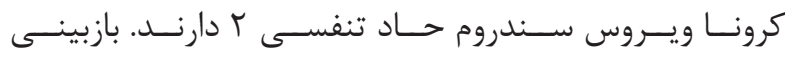

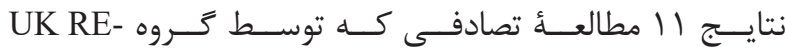

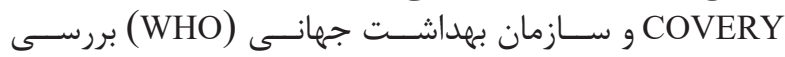

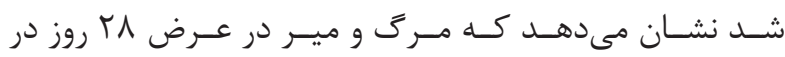

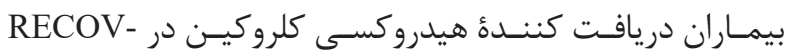

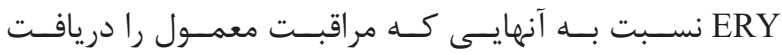

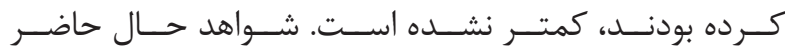

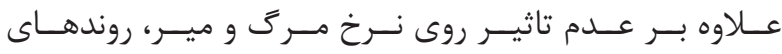

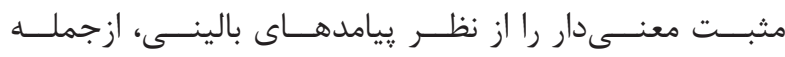

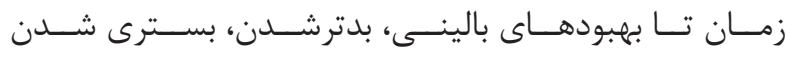

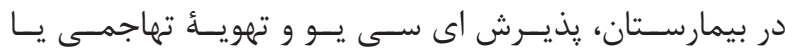

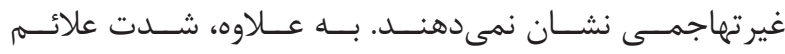

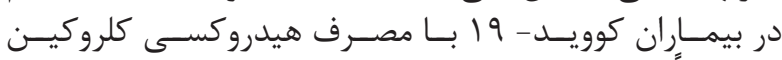

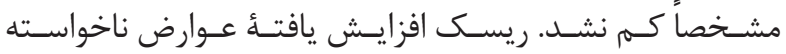

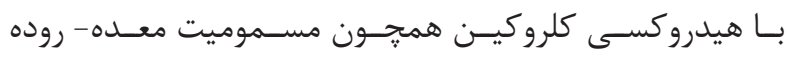

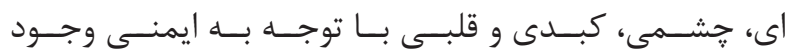

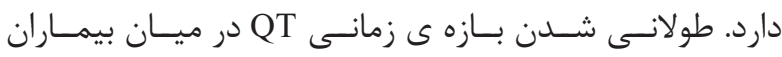

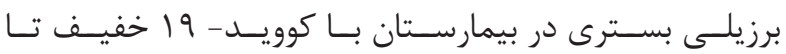

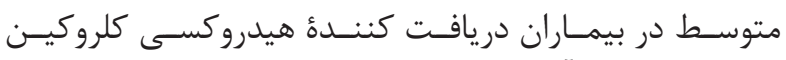

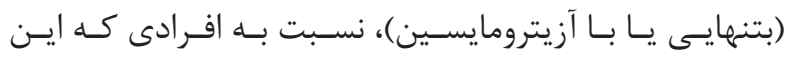

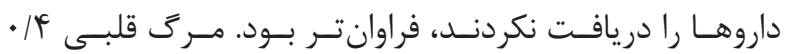




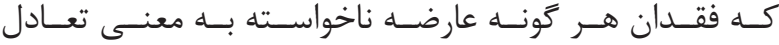

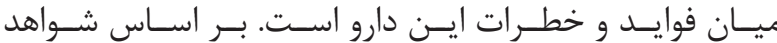

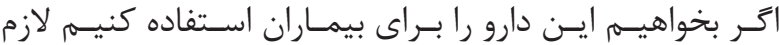

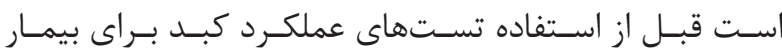

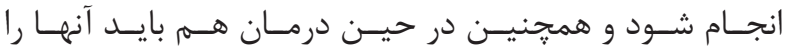

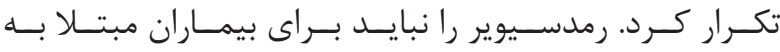

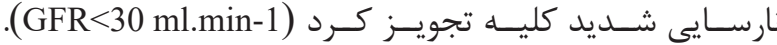

\section{ي ي}

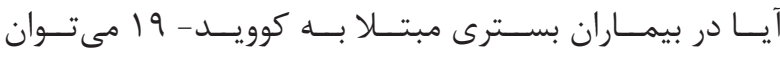

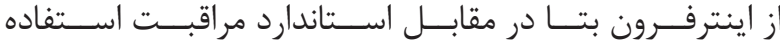

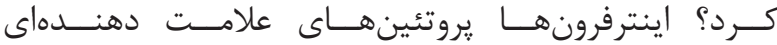

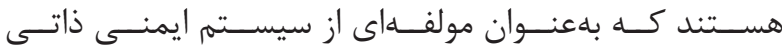

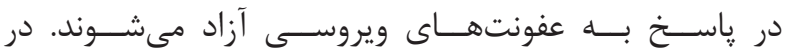

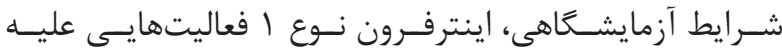

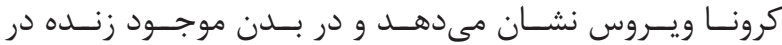

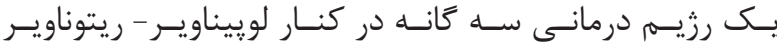

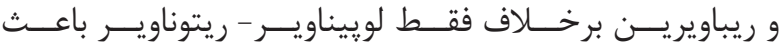

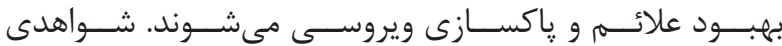

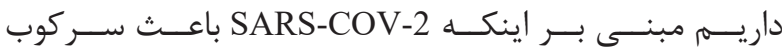

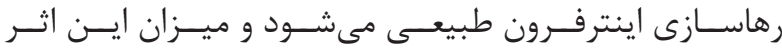

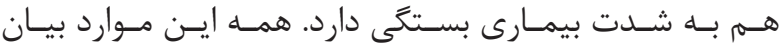

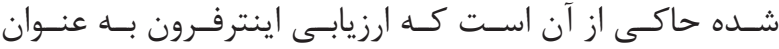

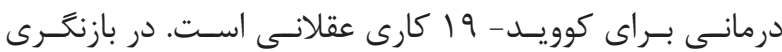

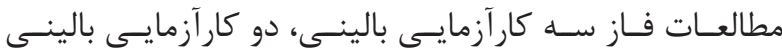

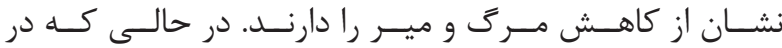

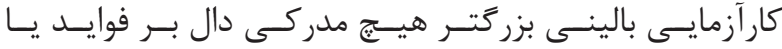

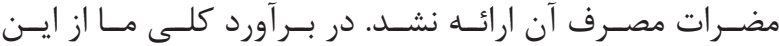

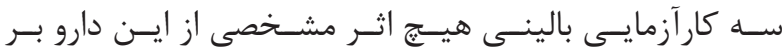

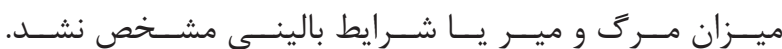

ييكو 1

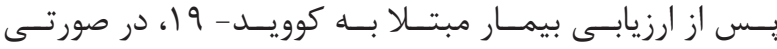

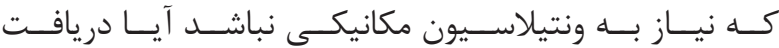

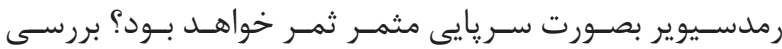

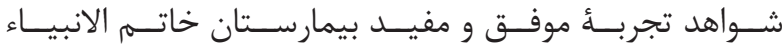

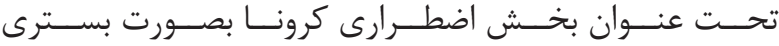

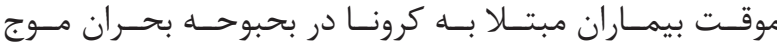

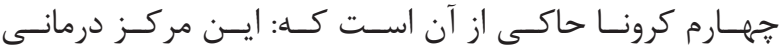

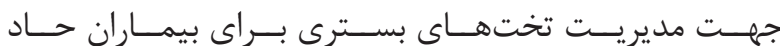

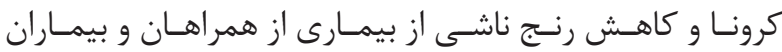

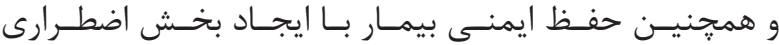

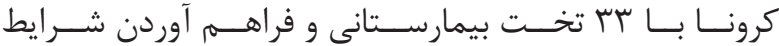

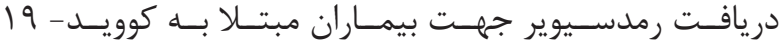

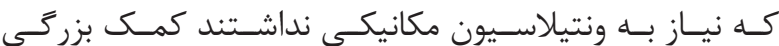

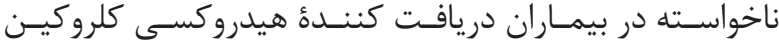

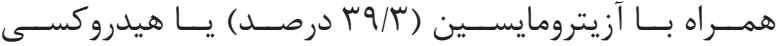

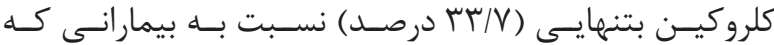

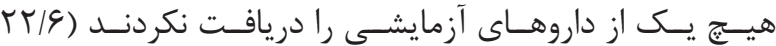

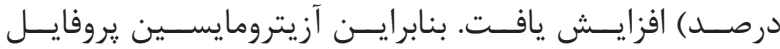

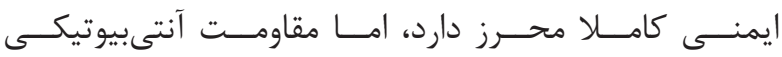

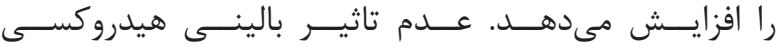

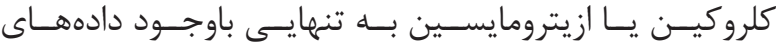

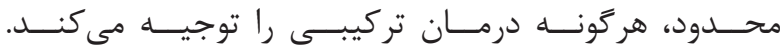

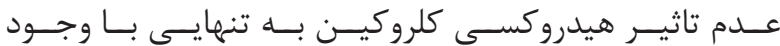

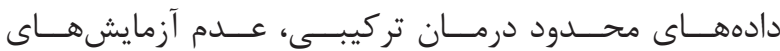

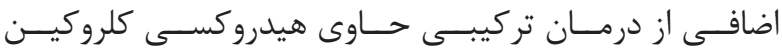

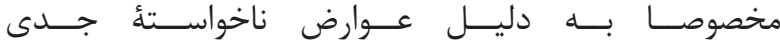

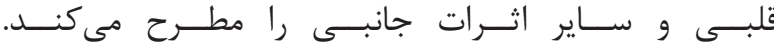

بيكو 9

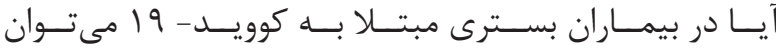

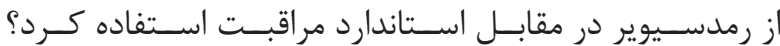

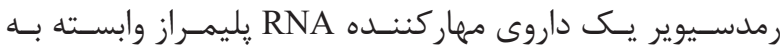

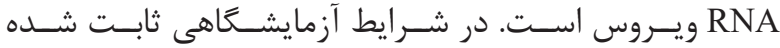

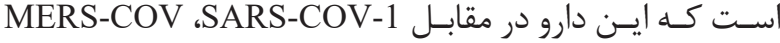

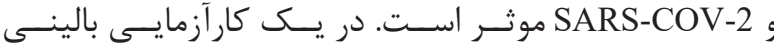

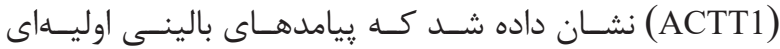

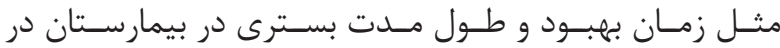

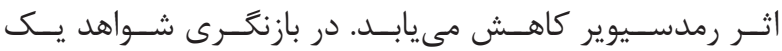

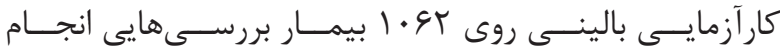

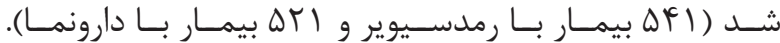

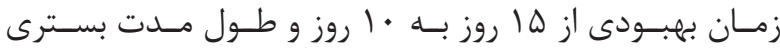

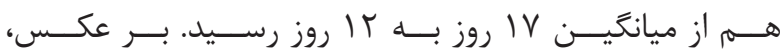

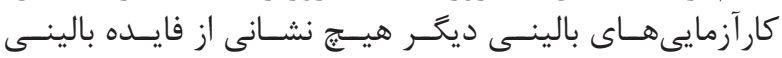

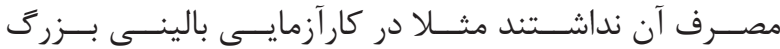

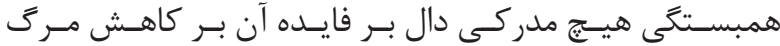

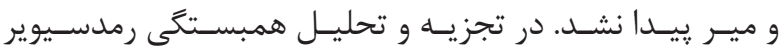

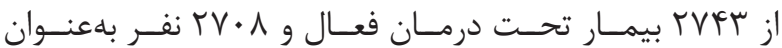

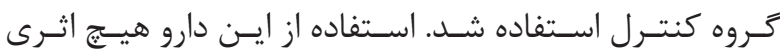

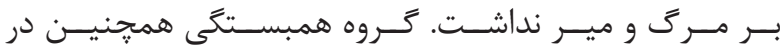

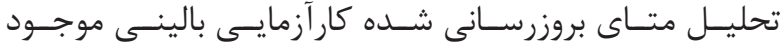

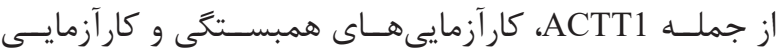

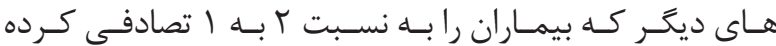

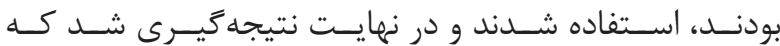

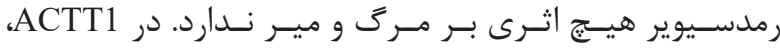

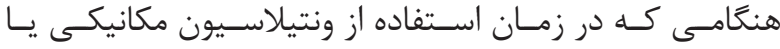

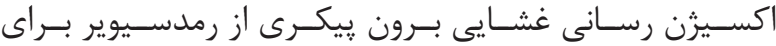

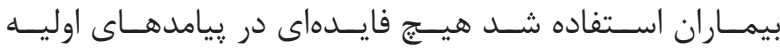

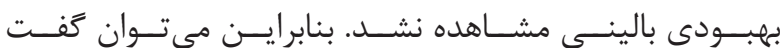




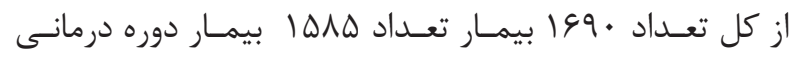

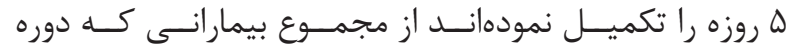

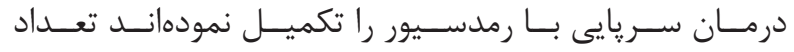

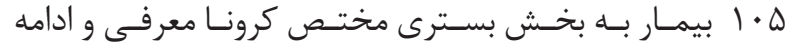

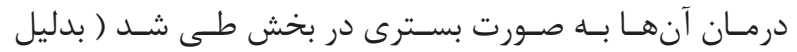

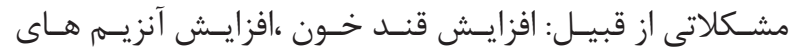

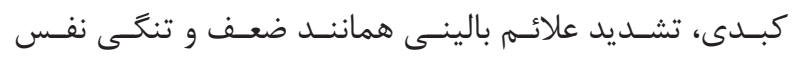

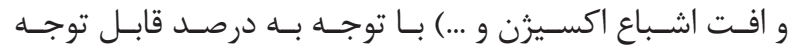

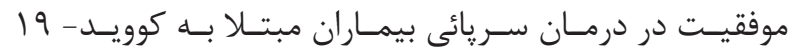

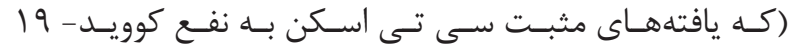

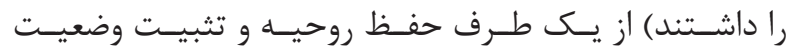

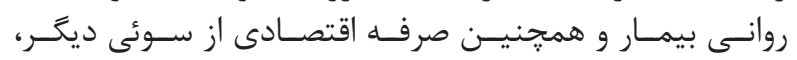

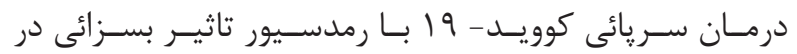

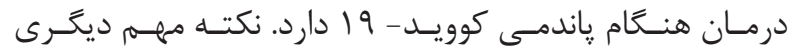

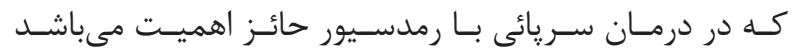

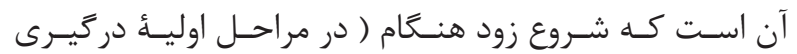

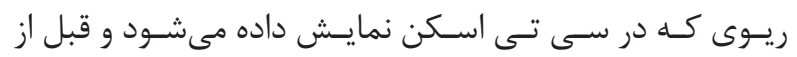

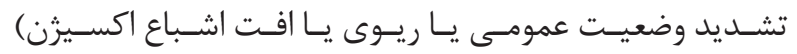

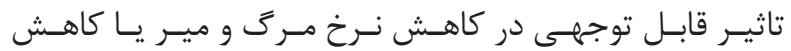

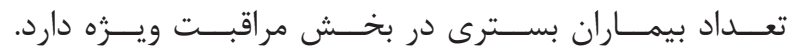

1. Deng Y, Liu W, Liu K, Fang Y-Y, Shang J, Zhou L, et al. Clinical characteristics of fatal and recovered cases of coronavirus disease2019in Wuhan, China:aretrospective study. Chinese medical journal. 2020; 133(11): 1261-7.

2. Guan $\mathrm{W}-\mathrm{j}$, Ni Z-y, Hu Y, Liang W-h, Ou $\mathrm{C}-\mathrm{q}$, He J-x, et al. Clinical characteristics of coronavirus disease 2019 in China. New England journal of medicine. 2020; 382(18): 1708-20.

3. Koh HK, Geller AC, VanderWeele TJ. Deaths from COVID-19. JAMA. 2021; 325(2): 133-4.

4. Ghinai I, McPherson TD, Hunter JC, Kirking HL, Christiansen D, Joshi K, et al. First known person-to-person transmission of severe acute respiratory syndrome coronavirus 2 (SARS-CoV-2) in the USA. The Lancet. 2020; 395(10230): 1137-44.

5. Liang W-h, Guan $\mathrm{W}-\mathrm{j}$, Li C-c, Li Y-m, Liang H-r, Zhao Y, et al. Clinical characteristics and outcomes of hospitalised patients with COVID-19 treated in Hubei (epicentre) and outside Hubei (non-epicentre): a nationwide analysis of China. European Respiratory Journal. 2020; 55(6): 2000562; DOI: 10.1183/13993003.00562-2020

6. Bai Y, Yao L, Wei T, Tian F, Jin D-Y, Chen L,

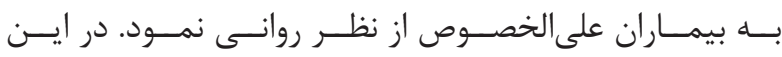

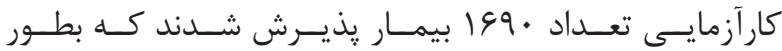

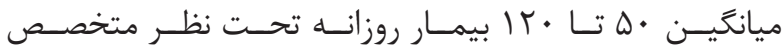

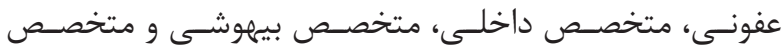

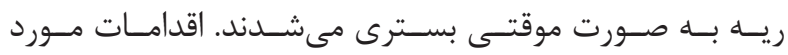

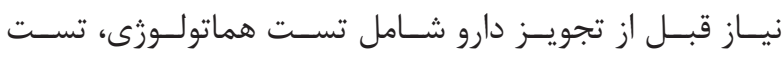

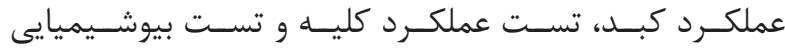

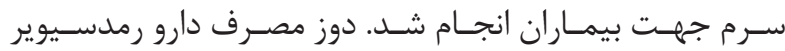

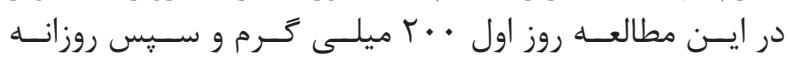

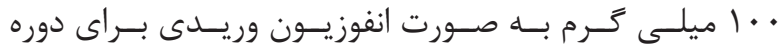

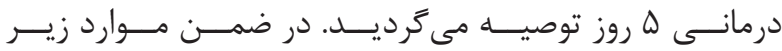

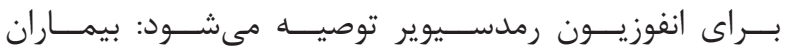

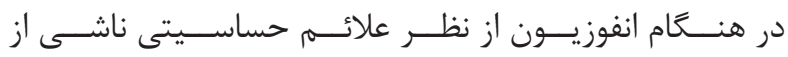

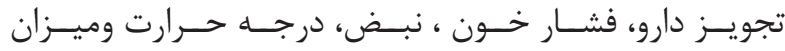

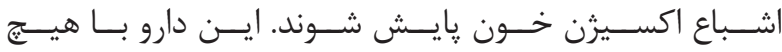

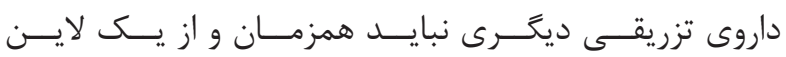

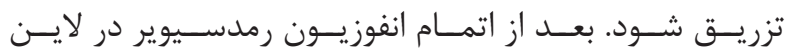

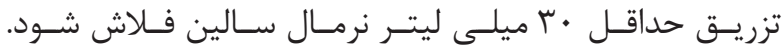
بحث و نتيجه تيرى

منابع et al. Presumed asymptomatic carrier transmission of COVID-19. Jama. 2020; 323(14): 1406-7.

7. Acosta MAT, Singer BD. Pathogenesis of COVID19-induced ARDS: implications for an ageing population. European Respiratory Journal. 2020; 56(3): 2002049; DOI: 10.1183/13993003.02049-2020

8. Nishiura H, Linton NM, Akhmetzhanov AR. Serial interval of novel coronavirus (COVID-19) infections. Internationaljournalofinfectiousdiseases. 2020;93:284-6.

9. Fan G, Tu C, Zhou F, Liu Z, Wang Y, Song B, et al. Comparison of severity scores for COVID-19 patients with pneumonia: a retrospective study. European Respiratory Journal. 2020; 56(3): 2002113; DOI: $\quad 10.1183 / 13993003.02113-2020$

10. Zhou F, Yu T, Du R, Fan G, Liu Y, Liu Z, et al. Clinical course and risk factors for mortality of adult inpatients with COVID-19 in Wuhan, China: a retrospective cohort study. The lancet. 2020; 395(10229): 1054-62.

11. Knight SR, Ho A, Pius R, Buchan I, Carson G, Drake TM, et al. Risk stratification of patients admitted to hospital with covid-19 using the ISARIC WHO Clinical Characterisation Protocol: development and validation of the 4C Mortality Score. bmj. 2020; 370. 
12. Du R-H, Liang L-R, Yang C-Q, Wang W, Cao T-Z, Li M, et al. Predictors of mortality for patients with COVID-19 pneumonia caused by SARSCoV-2: a prospective cohort study. European Respiratory Journal. 2020; 55(5): 2000524.

13. Docherty AB, Harrison EM, Green CA, Hardwick HE, Pius R, Norman L, et al. Features of 20133 UK patients in hospital with covid-19 using the ISARIC WHO Clinical Characterisation Protocol: prospective observational cohort study. bmj. 2020; 369.

14. Cevik M, Tate M, Lloyd O, Maraolo AE, Schafers J, Ho A. SARS-CoV-2, SARS-CoV, and MERS-CoV viral load dynamics, duration of viral shedding, and infectiousness: a systematic review and meta-analysis. The Lancet Microbe. 2020.

15. Swann OV, Holden KA, Turtle L, Pollock L, Fairfield CJ, Drake TM, et al. Clinical characteristics of children and young people admitted to hospital with covid-19 in United Kingdom: prospective multicentre observational cohort study. bmj. 2020; 370.

16. Wu G, Yang P, Xie Y, WoodruffHC, Rao X, Guiot J, et al. Development of a clinical decision support system for severity risk prediction and triage of COVID-19 patients at hospital admission: an international multicentre study. European Respiratory Journal. 2020; 56(2): 2001104.

17. Mason RJ. Pathogenesis of COVID-19 from a cell biology perspective. Eur Respiratory Soc; 2020.

18. Laing AG, Lorenc A, Del Barrio IDM, Das A, Fish M, Monin L, et al. A dynamic COVID-19 immune signature includes associations with poor prognosis. Nature medicine. 2020; 26(10): 1623-35.

19. Siddiqi HK, Mehra MR. COVID-19 illness in native and immunosuppressed states: a clinical-therapeutic staging proposal. The Journal of Heart and Lung Transplantation. 2020; 39(5): 405.

20. Del Valle DM, Kim-Schulze S, Huang H-H, Beckmann ND, Nirenberg S, Wang B, et al. An inflammatory cytokine signature predicts COVID-19 severity and survival. Nature medicine. 2020; 26(10): 1636-43.

21. Remy KE, Mazer M, Striker DA, Ellebedy AH, Walton AH, Unsinger J, et al. Severe immunosuppression and not a cytokine storm characterizes COVID-19 infections. JCI insight. 2020; 5(17): e140329.

22. Group RC. Effect of hydroxychloroquine in hospitalized patients with Covid-19. New England Journal of Medicine. 2020; 383(21): 2030-40.

23. Annane D. Corticosteroids for COVID-19. Journal of Intensive Medicine. 2021.

24. Diseases TLI. Curing COVID-19. The Lancet Infectious Diseases. 2020; 20(10): 1101. 